\title{
AN ALMOST EVERYWHERE EXISTENCE THEOREM FOR SOLUTIONS OF VOLTERRA FUNCTIONAL EQUATIONS( ${ }^{1}$ )
}

\author{
BY \\ J. YEH
}

1. Introduction. Let $C_{w}$ be the Wiener space, i.e., the collection of real valued functions $x(t)$ defined and continuous on $I: 0 \leqq t \leqq 1$ and satisfying $x(0)=0$.

Let a finite sequence of real valued continuous functions $F^{1}(t, u)$, $F^{2}\left(t, u, v_{1}\right), \cdots, F^{n}\left(t, u, v_{1}, \cdots, v_{n-1}\right)$ be defined and continuous for $t \in I$ and other variables unrestricted. The Volterra functionals $\Phi^{k}(x \mid t), \Lambda^{k}(x \mid t)$ depending on the function $x(\cdot)$ and the real variable $t$ are defined inductively by

$$
\begin{array}{rlrl}
\Lambda^{0}(x \mid t) & =x(t), & \text { on } C_{w} \otimes I, \\
\Phi^{k}(x \mid t)=F^{k}\left(t, \Lambda^{0}, \cdots, \Lambda^{k-1}\right), & & (k=1,2, \cdots, n) \text { on } C_{w} \otimes I, \\
\Lambda^{k}(x \mid t)=\int_{0}^{t} \Phi^{k}(\tau) d \tau & (k=1,2, \cdots, n) \text { on } C_{w} \otimes I .
\end{array}
$$

For any $x \in C_{w}$, the function $y$ defined by the Volterra functional equation

$$
y(t)=x(t)+\Lambda^{n}(x \mid t)
$$

or with $f=F^{n}$

$$
y(t)=x(t)+\int_{0}^{t} f\left[s, \Lambda^{0}(x \mid s), \cdots, \Lambda^{n-1}(x \mid s)\right] d s
$$

belongs to $C_{w}$. In [3] we showed that under certain conditions on $F^{k}$ there exists uniquely $x \in C_{w}$ satisfying (5) for every given $y \in C_{w}$. In the present article we prove an almost everywhere existence theorem for solutions of (5) where the phrase almost everywhere refers to the Wiener measure defined on $C_{w}$. Our result is the following:

Theorem. Let $F^{1}(t, u), F^{2}\left(t, u, v_{1}\right), \cdots, F^{n-1}\left(t, u, v_{1}, \cdots, v_{n-2}\right)$, $f\left(t, u, v_{1}, \cdots, v_{n-1}\right)$ be continuous and have continuous first derivatives with respect to $u, v_{1}, \cdots, v_{n-1}$ on $I \otimes R_{k}(k=1,2, \cdots, n)$ where $R_{k}$ is the $k$-dimensional Euclidean space and let $f_{t}$ be continuous on $I \otimes R_{n}$. Let $F^{1}, F^{2}, \cdots, F^{n-1}$,

Received by the editors August 21, 1961.

(1) The author is indebted to Professor R. H. Cameron for his advice in writing this paper. 
$f$ satisfy the order of growth conditions $\left({ }^{2}\right)$

(6) $\left|F^{k}\left(t, u, v_{1}, \cdots, v_{k-1}\right)\right| \leqq C \sum_{j=0}^{k-1}\left|v_{j}\right|$ on $I \otimes R_{k}(k=1,2, \cdots, n-1)$,

(7) $f\left(t, u, v_{1}, \cdots, v_{n-1}\right) \operatorname{sgn} u \geqq-A_{1}\{B u\}^{2}$ on $I \otimes R_{n}$,

(8) $\quad f_{u}+4 g_{t}+4 \sum_{j=1}^{n-1} g_{j} F^{j} \leqq 2 \sum_{j=0}^{n-1} \alpha_{j}^{2} v_{j}^{2}+A_{2}$ on $I \otimes R_{n}$,

(9) $\quad g\left(1, u, v_{1}, \cdots, v_{n-1}\right) \geqq-\frac{1}{2} \alpha(\cot \beta) u^{2}-A_{3}$ on $R_{n}$,

where

$$
\begin{aligned}
g\left(t, u, v_{1}, \cdots, v_{n-1}\right) & =\int_{0}^{u} f\left(t, u^{\prime}, v_{1}, \cdots, v_{n-1}\right) d u^{\prime} \quad \text { on } I \otimes R_{n} \\
\alpha & =\left\{\alpha_{0}^{2}+\sum_{j=1}^{n-1}\left[C(1+C)^{j-1}\right]^{2} \alpha_{j}^{2}\right\}^{1 / 2}
\end{aligned}
$$

and $A_{1}, A_{2}, B, C, \alpha_{j}(j=0,1, \cdots, n-1), \beta$ are positive constants satisfying $\alpha<\beta<\pi$ and $B<1$. Then corresponding to almost every $y \in C_{w}$, (5) has a solution $x \in C_{w}$ which is unique in $C_{w}$.

2. Lemma. Let each of $F^{1}(t, u), F^{2}\left(t, u, v_{1}\right), \cdots$ be continuous and satisfy (6) on $I \otimes R_{k},(k=1,2, \cdots)$. Then for any $x \in C_{w}$ the Volterra functional $\Lambda^{k}(x \mid t),(k=0,1,2, \cdots)$ defined by (1), (2), (3) satisfies

$$
\Lambda^{k}(x \mid t) \leqq C(1+C)^{k-1}\left\{\int_{0}^{t}[x(s)]^{2} d s\right\}^{1 / 2}
$$

for $t \in I,(k=1,2, \cdots)$.

Proof. The proof is based on Schwarz's inequality and a complete induction on $k$. For $k=1$, by (6) and Schwarz's inequality

$$
\begin{aligned}
\left|\Lambda^{1}(x \mid t)\right| & \leqq \int_{0}^{t}\left|F^{1}[s, x(s)]\right| d s \\
& \leqq C \int_{0}^{t}|x(s)| d s \\
& \leqq C\left\{\int_{0}^{t}[x(s)]^{2} d s\right\}^{1 / 2} \quad \text { for } t \in I .
\end{aligned}
$$

Now suppose that (12) holds for $1 \leqq k \leqq N$. Then again by (6) and Schwarz's inequality

(2) $v_{0}=u$. 


$$
\begin{aligned}
\left|\Lambda^{N+1}(x \mid t)\right| \leqq & \int_{0}^{t}\left|F^{N+1}\left[s, x(s), \Lambda^{1}(x \mid s), \cdots, \Lambda^{N}(x \mid s)\right]\right| d s \\
\leqq & C \int_{0}^{t}|x(s)| d s+C \sum_{j=1}^{N} \int_{0}^{t}\left|\Lambda^{j}(x \mid s)\right| d s \\
\leqq & C\left\{\int_{0}^{t}[x(s)]^{2} d s\right\}^{1 / 2} \\
& +C \sum_{j=1}^{N} \int_{0}^{t} C(1+C)^{j-1}\left\{\int_{0}^{s}[x(r)]^{2} d r\right\}^{1 / 2} d s \\
\leqq & \left.C 1+C \sum_{j=1}^{N}(1+C)^{j-1}\right\}\left\{\int_{0}^{t}[x(s)]^{2} d s\right\}^{1 / 2} \\
= & C(1+C)^{N}\left\{\int_{0}^{t}[x(s)]^{2} d s\right\}^{1 / 2}
\end{aligned}
$$

and (12) holds for $k=N+1$ as well. This completes the proof of (12) by induction.

3. Proof of the theorem. Let $\gamma=B^{-1}-1>0$ and

$$
\phi(t, u)=(t+\gamma)^{-1 / 2} \exp \left\{(t+\gamma)^{-1} u^{2}\right\}
$$

on $I \otimes R_{1}$.

Since $t+\gamma>0$ on $I, \phi(t, u)$ has continuous derivatives of all orders with respect to $t$ and $u$ on $I \otimes R_{1}$. Define a function $G\left(t, u, v_{1}, \cdots, v_{n-1}, \lambda\right)$ depending on a non-negative parameter $\lambda$ by

$$
G\left(t, u, v_{1}, \cdots, v_{n-1} \mid \lambda\right)=g\left(t, u, v_{1}, \cdots, v_{n-1}\right)+\lambda \phi(t, u)
$$

Then

$$
\text { on } I \otimes R_{n}, \lambda \geqq 0 \text {. }
$$

$$
\begin{aligned}
G_{t}=g_{t}-\frac{1}{2} \lambda(t+\gamma)^{-1} \phi(t, u)-\lambda(t+\gamma)^{-2} u^{2} \phi(t, u), & \\
G_{u}=f+2 \lambda(t+\gamma)^{-1} u \phi(t, u), & \text { on } I \otimes R_{n}, \lambda \geqq 0, \\
G_{j}=g_{j},(j=1,2, \cdots, n-1) & \text { on } I \otimes R_{n}, \lambda \geqq 0,
\end{aligned}
$$

and these derivatives are all continuous. Furthermore $G_{u}$ has continuous first derivatives with respect to $t, u, v_{1}, \cdots, v_{n-1}$ on $I \otimes R_{n}$ for $\lambda \geqq 0$ and in particular $G_{u u}$ is given by

$$
G_{u u}=f_{u}+2 \lambda(t+\gamma)^{-1} \phi(t, u)+4 \lambda(t+\gamma)^{-2} u^{2} \phi(t, u)
$$

so that by (15)

$$
G_{u u}+4 G_{t}=f_{u}+4 g_{t} \quad \text { on } I \otimes R_{n} \text { for } \lambda \geqq 0 .
$$

Now $F^{1}, F^{2}, \cdots, F^{n-1}, G_{u}$ satisfy the conditions on $F^{1}, F^{2}, \cdots, F^{n-1}, f$ of Theorem 1 of [2] so that the transformation 


$$
y(t)=x(t)+\int_{0}^{t} G_{u}\left[s, \Lambda^{0}(x \mid s), \cdots, \Lambda^{n-1}(x \mid s) \mid \lambda\right] d s, \quad \lambda \geqq 0
$$

transforms $C_{w}$ in a 1-1 manner into a measurable subset $\Gamma$ with a measure given by $\left.{ }^{3}\right)$

$$
m_{w}(\Gamma)=\int_{c_{w}} \exp \{J(x, \lambda)\} d_{w} x, \quad \lambda \geqq 0
$$

where

$$
\begin{aligned}
J(x, \lambda)= & \int_{0}^{1} K\left[t, \Lambda^{0}(x \mid t), \cdots, \Lambda^{n-1}(x \mid t) \mid \lambda\right] d t \\
& +2 G(0,0, \cdots, 0 \mid \lambda)-2 G\left[1, \Lambda^{0}(x \mid 1), \cdots, \Lambda^{n-1}(x \mid 1) \mid \lambda\right]
\end{aligned}
$$

for $x \in C_{w}, \lambda \geqq 0$ with

$$
\begin{aligned}
K\left(l, u, v_{1}, \cdots v_{n-1} \mid \lambda\right) & =\frac{1}{2} G_{u u}+2 G_{t}-G_{u}^{2}+2 \sum_{j=1}^{n-1} G_{j} F^{j} \\
& =\frac{1}{2} f_{u}+2 g_{t}-G_{u}^{2}+2 \sum_{j=1}^{n-1} g_{j} F^{j}, \quad \text { for } \lambda \geqq 0
\end{aligned}
$$

according to (19), (17).

We show next that for each positive value of $\lambda$ the transformation (20) transforms $C_{w}$ 1-1 onto itself. We only have to show that when $\lambda>0, G_{u}$ satisfies the condition (4.1) of Theorem II of [3]. From(16)

$$
G_{u} \operatorname{sgn} u=f \operatorname{sgn} u+2 \lambda(t+\gamma)^{-1}|u| \phi(t, u) \quad \text { on } I \otimes R_{n} \text { for } \lambda \geqq 0
$$

and from (13) and $0<t+\gamma \leqq 1+\gamma,(t+\gamma)^{-1} \geqq B$ for $t \in I$,

$$
\begin{aligned}
2 \lambda(t+\gamma)^{-1}|u| \phi(t, u) & =2 \lambda(t+\gamma)^{-3 / 2}|u| \exp \left\{(t+\gamma)^{-1} u^{2}\right\} \\
& \geqq 2 \lambda B^{3 / 2}|u| \exp \left\{B u^{2}\right\}, \quad \text { on } I \otimes R_{1} \text { for } \lambda \geqq 0 .
\end{aligned}
$$

Now when $\lambda>0$ and $|u| \geqq \lambda^{-1} B^{-3 / 2} A_{1},(25)$ implies

$$
2 \lambda(t+\gamma)^{-1}|u| \phi(t, u) \geqq 2 A_{1} \exp \left\{B u^{2}\right\}
$$

so that according to (24), (7), (26)

$$
G_{u} \operatorname{sgn} u \geqq A_{1} \exp \left\{B u^{2}\right\} \geqq A_{1}
$$

for $\lambda>0,|u| \geqq \lambda^{-1} B^{-3 / 2} A_{1},\left(t, v_{1}, v_{2}, \cdots, v_{n-1}\right) \in I \otimes R_{n-1}$. On the other hand when $\lambda>0$ but $|u| \leqq A^{-1} B^{-3 / 2} A_{1}$,

(3) See the first equation on p. 152 of [2]. 


$$
f \operatorname{sgn} u \geqq-A_{1} \exp \left\{B \lambda^{-2} B^{-3} A_{1}^{2}\right\}=-A(\lambda)
$$

according to (7) where by definition

$$
A(\lambda)=A_{1} \exp \left\{\lambda^{-2} B^{-2} A_{1}^{2}\right\}>0
$$

and hence by (24), (25)

$$
G_{u} \operatorname{sgn} u \geqq-A(\lambda)
$$

for $\lambda>0,|u| \leqq \lambda^{-1} B^{-3 / 2} A_{1},\left(t, v_{1}, v_{2}, \cdots, v_{n-1}\right) \in I \otimes R_{n-1}$.

Summarizing (27), (28) we obtain

$$
G_{u} \operatorname{sgn} u \geqq-A(\lambda) \quad \text { on } I \otimes R_{n} \text { with } A(\lambda)>0 \text { for } \lambda>0 .
$$

Thus for each $\lambda>0, G_{u}$ satisfies (4.1) of [3] and according to Theorem II of [3] the transformation (20) transforms $C_{w} 1-1$ onto itself. From (21) we have

$$
1=\int_{c_{w}} \exp \{J(x, \lambda)\} d_{w} x \quad \text { for } \lambda>0 .
$$

Now since $G_{u}\left(t, u, v_{1}, \cdots, v_{n-1} \mid 0\right)=f\left(t, u, v_{1}, \cdots, v_{n-1}\right)$ according to (16), we only have to show that (30) holds even when $\lambda=0$ in order to complete the proof of the theorem. We show

$$
\lim _{\lambda \downarrow 0} \int_{C_{w}} \exp \{J(x, \lambda)\} d_{w} x=\int_{C_{w}} \exp \{J(x, 0)\} d_{w} x .
$$

This is done in what follows by interchanging the order of integration and limiting process.

According to (22), (14), for each $x \in C_{w}$

$$
\begin{aligned}
\lim _{\lambda \downarrow 0} J(x, \lambda)= & \lim _{\lambda \downarrow 0}\left\{\int_{0}^{1} K\left[t, \Lambda^{0}(x \mid t), \cdots, \Lambda^{n-1}(x \mid t) \mid \lambda\right] d t\right\} \\
& -2 g(0,0, \cdots, 0)-2 g\left[1, \Lambda^{0}(x \mid 1), \cdots, \Lambda^{n-1}(x \mid 1)\right] .
\end{aligned}
$$

To pass to the limit under the integral sign in (32) we show that for each fixed $x \in C_{w}, K$ is bounded on $I$ for $0<\lambda \leqq 1$. From (23), (16), (13)

$$
\begin{aligned}
& K[t,\left.\Lambda^{0}(x \mid t), \cdots, \Lambda^{n-1}(x \mid t) \mid \lambda\right] \\
&=\frac{1}{2} f_{u}\left[t, \Lambda^{0}(x \mid t), \cdots, \Lambda^{n-1}(x \mid t)\right]+2 g_{t}\left[t, \Lambda^{0}(x \mid t), \cdots, \Lambda^{n-1}(x \mid t)\right] \\
&-\left\{f\left[t, \Lambda^{0}(x \mid t), \cdots, \Lambda^{n-1}(x \mid t)\right]+2 \lambda(t+\gamma)^{-3 / 2} \Lambda^{0}(x \mid t)\right. \\
&\left.\cdot \exp \left\{(t+\gamma)^{-1}\left[\Lambda^{0}(x \mid t)\right]^{2}\right\}\right\}^{2} \\
&+2 \sum_{j=1}^{n-1} g_{j}\left[t, \Lambda^{0}(x \mid t), \cdots, \Lambda^{n-1}(x \mid t)\right] F^{j}\left[t, \Lambda^{0}(x \mid t), \cdots, \Lambda^{n-1}(x \mid t)\right] \\
& \quad \text { for } t \in I, \lambda \geqq 0 .
\end{aligned}
$$


From the continuity of $F^{1}, F^{2}, \cdots, F^{n-1}, f, f_{u}, g_{t}, g_{j}(j=1,2, \cdots, n-1)$ it is evident that $K$ is bounded on $I$ for $0<\lambda \leqq 1$ for each $x \in C_{w}$. Also from (23), (16)

$$
\begin{aligned}
\lim _{\lambda \downarrow 0} K\left(t, u, v_{1}, \cdots, v_{n-1} \mid \lambda\right) & =\frac{1}{2} f_{u}+2 g_{t}-f^{2}+2 \sum_{j=1}^{n-1} g_{j} F^{i} \\
& =K\left(t, u, v_{1}, \cdots, v_{n-1} \mid 0\right)
\end{aligned}
$$

and from (32), (22), (14)

$$
\begin{aligned}
\lim _{\lambda \downarrow 0} J(x, \lambda)= & \int_{0}^{1} K\left[t, \Lambda^{0}(x \mid t), \cdots, \Lambda^{n-1}(x \mid t) \mid 0\right] d t-2 g(0,0, \cdots, 0) \\
& -2 g\left[1, \Lambda^{0}(x \mid 1), \cdots, \Lambda^{n-1}(x \mid 1)\right]=J(x, 0) .
\end{aligned}
$$

We next justify

$$
\lim _{\lambda \downarrow 0} \int_{C_{w}} \exp \{J(x, \lambda)\} d_{w} x=\int \lim _{C_{w}} \exp \{J(x, \lambda)\} d_{w} x
$$

by dominating $\exp \{J(x, \lambda)\}$ on $C_{w}$ for all $\lambda>0$ by a function which is independent of $\lambda$ and integrable on $C_{w}$. From (22), (23), (14), (13)

$$
\begin{aligned}
J(x, \lambda) \leqq & \int_{0}^{1}\left\{\frac{1}{2} f_{u}\left[t, \Lambda^{0}, \cdots, \Lambda^{n-1}\right]+2 g_{t}\left[t, \Lambda^{0}, \cdots, \Lambda^{n-1}\right]\right. \\
& \left.+2 \sum_{j=1}^{n-1} g_{j}\left[t, \Lambda^{0}, \cdots, \Lambda^{n-1}\right] F^{j}\left[t, \Lambda^{0}, \cdots, \Lambda^{j-1}\right]\right\} d t \\
& -\int_{0}^{1}\left\{G_{u}\left[t, \Lambda^{0}, \cdots, \Lambda^{n-1} \mid \lambda\right]\right\}^{2} d t \\
& +2\left\{g(0,0, \cdots, 0)+\lambda \gamma^{-1 / 2}\right\} \\
& -2\left\{g\left[1, \Lambda^{0}(1), \cdots, \Lambda^{n-1}(1)\right]+\lambda B^{1 / 2} \exp \left\{B\left[\Lambda^{0}(1)\right]^{2}\right\}\right\} .
\end{aligned}
$$

The second integral in the right-hand side is non-negative. Also $g(0,0, \cdots, 0)$ $=0$ by (10), and $\lambda B^{1 / 2} \exp \left\{B\left[\Lambda^{0}(1)\right]^{2}\right\}>0$. Therefore when $1>\lambda>0$

$$
\begin{aligned}
J(x, \lambda) \leqq & \int_{0}^{1}\left\{\frac{1}{2} f_{u}\left[t, \Lambda^{0}, \cdots, \Lambda^{n-1}\right]+2 g_{t}\left[t, \Lambda^{0}, \cdots, \Lambda^{n-1}\right]\right. \\
& \left.+2 \sum_{j=1}^{n-1} g_{j}\left[t, \Lambda^{0}, \cdots, \Lambda^{n-1}\right] F^{j}\left[t, \Lambda^{0}, \cdots, \Lambda^{j-1}\right]\right\} d t \\
& -2 g\left[1, \Lambda^{0}(1), \cdots, \Lambda^{n-1}(1)\right]+2 \gamma^{-1 / 2} \quad \text { for all } x \in C_{w} .
\end{aligned}
$$

The right-hand side of (36) is independent of $\lambda$. By (8), (9), Lemma, (11) 


$$
\begin{aligned}
J(x, \lambda) \leqq & \int_{0}^{1}\left\{\sum_{j=0}^{n-1} \alpha_{j}^{2}\left[\Lambda^{j}(x \mid t)\right]^{2}+\frac{A_{2}}{2}\right\} d t+\alpha \cot \beta\left[\Lambda^{0}(x \mid 1)\right]^{2}+2 A_{3}+2 \gamma^{-1 / 2} \\
\leqq & \alpha_{0}^{2} \int_{0}^{1}[x(t)]^{2} d t+\int_{0}^{1} \sum_{j=1}^{n-1} \alpha_{j}^{2}\left[C(1+C)^{j-1}\right]^{2}\left\{\int_{0}^{t}[x(s)]^{2} d s\right\} d t \\
& +\frac{A_{2}}{2}+\alpha \cot \beta[x(1)]^{2}+2 A_{3}+2 \gamma^{-1 / 2} \\
\leqq & \alpha^{2}\left\{\int_{0}^{1}[x(t)]^{2} d t\right\}+\frac{A_{2}}{2}+\alpha \cot \beta[x(1)]^{2}+2 A_{3}+2 \gamma^{-1 / 2}
\end{aligned}
$$

and

$$
\begin{aligned}
\exp \{J(x, \lambda)\} \leqq & \exp \left\{\alpha^{2} \int_{0}^{1}[x(t)]^{2} d t+\alpha \cot \beta[x(1)]^{2}\right\} \\
& \cdot \exp \left\{\frac{A_{2}}{2}+2 A_{3}+2 \gamma^{-1 / 2}\right\} .
\end{aligned}
$$

According to $\$ 2$ of [1], the right-hand side of (37) is integrable on $C_{w}$. Thus (35) is valid, (31) is valid by (34) and (30) holds for $\lambda=0$, which means that for almost every $y \in C_{w}$, (5) has a solution $x \in C_{w}$. Its uniqueness follows from Remark 1, §2 of [3].

4. An example. We give an example with $n=2$ to which the present almost everywhere existence theorem is applicable but not the everywhere existence theorem, Theorem II of [3]. Let

$$
\begin{aligned}
F^{1}(t, u) & =\sin u, \\
f(t, u, v) & =\frac{1}{10}\left(u^{2} \sin 2 u+2 u \sin ^{2} u\right) \sin ^{2} v .
\end{aligned}
$$

Then

$$
\begin{aligned}
f_{u}(t, u, v) & =\frac{1}{10}\left(2 u^{2} \cos 2 u+4 u \sin 2 u+2 \sin ^{2} u\right) \sin ^{2} v \\
g(t, u, v) & =\frac{1}{10} u^{2} \sin ^{2} u \sin ^{2} v \\
g_{t}=0, g_{v} F^{1} & =\frac{1}{10} u^{2} \sin ^{3} u \sin 2 v
\end{aligned}
$$

so that 


$$
\begin{aligned}
\left|F^{1}(t, u)\right| & \leqq|u|, \\
|f(t, u, v)| & \leqq \frac{1}{10}\left(u^{2}+2|u|\right) \leqq \exp \left\{\frac{1}{2} u^{2}\right\} \\
f_{u}+4 g_{t}+ & 4 g_{v} F^{1} \\
& \leqq \frac{1}{10}\left(2 u^{2}+4|u|+2\right)+\frac{4}{10} u^{2} \leqq \frac{1}{10}\left(6 u^{2}+6\right)+\frac{4}{10} u^{2}=u^{2}+1, \\
g(1, u, v) & \geqq 0,
\end{aligned}
$$

and the conditions in the theorem are satisfied with $A_{1}=A_{2}=1, A_{3}>0$, $B=1 / 2, C \geqq 1, \alpha_{0}=1, \alpha_{1}=0, \alpha_{0}=1<\beta<\pi$. On the other hand (4.1) of [3] is violated.

\section{BIBLIOGRAPHY}

1. R. H. Cameron, Differential equations involving a parametric function, Proc. Amer. Math. Soc. 8 (1957), 834-840.

2. - Nonlinear Volterra functional equations and linear parabolic differential systems, J. Analyse Math. 5 (1956/1957), 136-182.

3. J. Yeh, Nonlinear Volterra functional equations and linear parabolic differential systems, Trans. Amer. Math. Soc. 95 (1960), 408-432.

UNIVERSITY OF ROCHESTER,

ROCHESTER, NEW YORK 